\title{
ESTUDIO DESCRIPTIVO DE FORMA Y FUNCION DEL JUEGO LIBRE DEL NIÑO(a) EN ETAPA PREESCOLAR.
}

\author{
DESCRIPTIVE STUDY ON THE FORM AND FUNCTION OF UNSTRUCTURED PLAY IN THE PRE- \\ SCHOOL CHILD.
}

\author{
Palma Candia, Oskarina ${ }^{1}$. Lavadie Saldivia, María Inés ${ }^{2}$. Paredes Ojeda, Cristian ${ }^{3}$ \\ Seguel Nahuelquín, Hernán ${ }^{4}$ Uribe Maldonado, Karina ${ }^{5}$
}

\section{Resumen}

La investigación tiene como principal objetivo conocer la forma y función del juego libre de 7 niños (as) en etapa de transición (4 a 5 años) de la ciudad de Punta Arenas, considerados en establecimientos educacionales pre-escolares en la región de Magallanes y Antártica Chilena. Bajo los fundamentos teóricos de la Ciencia de la Ocupación, se pretende identificar, a través del juego libre, aspectos relevantes de la conducta del menor, que favorezcan o dificulten su desempeño escolar. La metodología usada es de carácter cualitativo, siendo su método el estudio de caso. Los principales resultados demuestran similitud en la función del juego libre de cada niño(a), siendo estos predominantemente motor-social, los cuales repercuten en su desempeño escolar. Se concluye que, cada juego significa un momento para el aprendizaje y, como herramienta diagnóstica, entrega la posibilidad de visualizar ciertas conductas adaptativas en los niños(as) o, por el contrario, determinar signos de alerta según su edad de desarrollo.

\section{Palabras clave}

Ocupación, Juego libre, desempeño pre-escolar, forma y función.

\footnotetext{
${ }^{1}$ Terapeuta Ocupacional, Magister de gestión en salud, diplomada en docencia en Ciencias de la Salud, profesor asistente Universidad de Magallanes. oskarina.palma@umag.cl

${ }^{2}$ Licenciada en Ciencia de la Ocupación, UMAG. mane-75@hotmail.com

${ }^{3}$ Licenciada en Ciencia de la Ocupación,UMAG. nashito_97@hotmail.com

${ }^{4}$ Licenciada en Ciencia de la Ocupación, UMAG. ignora te@hotmail.com

${ }^{5}$ Licenciada en Ciencia de la Ocupación, UMAG. karis_ika@hotmail.com
} 


\section{Abstract}

The AIM of this research is to investigate the form and function of the unstructured play of seven children in transition stage ( 4 to 5 year-old) in the city of Punta Arenas. The children considered in this study are from preschool educational institutions living in the region of Magallanes and the Chilean Antartica. Under the theoretical foundations of Occupational Science it is expected to identify the relevant aspects of the children's behavior in unstructured play, especially those that may help, or having negative effects in the school performance.

The methodology used in this case is qualitative, being its method the case study. The main results demonstrate similarities regarding the functions of the children's unstructured play, being these mainly "motor-social" functions that have an effect in the school performance. It can be concluded then, that unstructured play helps to develop the children's learning strategies. It can be used as a diagnosis tool because it permits the observation of adaptive behaviors in children, and also allows determining signs of alert considering the child's age of development.

\section{Key words}

Occupation, Unstructured play, Preschool performance, Form and function. 


\section{INTRODUCCIÓN}

La Ocupación es una actividad inherente al ser humano que le otorga significado e identidad. Mediante ésta, el individuo satisface necesidades y deseos e interactúa con su entorno.( Christiansen, 1999) El juego como ocupación, es un medio placentero, en el cual el niño expresa diferentes pensamientos y emociones, es considerado no sólo como un sinónimo de recreación sino también como una modalidad terapéutica. Logrando a través de éste desempeños fundamentales para el crecimiento físico, intelectual, emocional y social del niño. En definitiva, el juego es una actividad primordial en su desarrollo psicomotor, que lo preparará a afrontar las exigencias de la sociedad en la vida adulta. Referido a esto, Polonio (2008) menciona que la Ocupación juego colabora en formar la identidad ocupacional del niño. Christiansen (1999) define el concepto de identidad como la expresión unificada de nuestro ser y el propósito en la vida. Los seres humanos expresan su identidad a través de las ocupaciones que dan significado a su existencia. En la niñez, esta ocupación es el juego (Polonio, 2008).

Dicha ocupación posee una forma, función y significado, donde cada una de ellas describe a la actividad, influenciándose mutuamente. Por un lado, la forma se refiere a las características directamente observables de la ocupación, las cuales pueden ser los aspectos notorios de diversas culturas, grupos, edades y estilos, incluyendo el contexto físico y los materiales que se utilizan para llevar a cabo cualquier actividad. Respecto a la función, ésta se refiere a la interacción dinámica entre la ocupación y el individuo, entregando un propósito personal a determinada actividad, sólo cuando alguien la realiza. Por último, se entiende por significado el valor subjetivo que construye cada persona al realizar alguna actividad, determinada por las experiencias en dichas ocupaciones (Willard, Crepeau, 2005).

Dentro de la etapa pre-escolar, el niño(a) suele ser un investigador natural inspirado principalmente por la curiosidad, y al mismo tiempo va desarrollando rápidamente una gama de destrezas que lo ayudarán a comprender mejor el mundo que lo rodea, según la etapa de desarrollo en la que se encuentren. Estas habilidades le permiten desempeñarse en sus ocupaciones y a futuro lo prepararán para las exigencias de un entorno lleno de barreras y oportunidades. Por otra parte el contexto donde se realizan los juegos favorece o dificulta la aparición de diversos desafíos que estimulan el desarrollo a través del desempeño ocupacional, se incluye en esto si son espacios cerrados o ambientes naturales y se considera los juguetes o elementos del ambientes que motivan el juego en el niño. En particular, si consideramos que nuestras ocupaciones ocurren en un contexto y que la mayoría de las ocupaciones son situadas fuertemente dentro del reino espacial ambiental, el "lugar" conlleva el potencial para influenciar fuertemente o dar forma al significado que nosotros obtenemos desde nuestras experiencias diarias que son conducidas a través de la ocupación.( Manuel, P.2003) 
Por contraparte un ambiente poco acogedor o estimulante dificultará la aparición de algunas habilidades, a su vez el contexto escolar direccionará ciertas habilidades que no son las mismas que pudiesen darse en un juego no estructurado.

La presente investigación, bajo la mirada de la Ciencia de la Ocupación, tiene como principal objetivo observar, describir y comprender la forma y función del juego libre de 7 niños(as) de distintos establecimientos pre-escolares en la ciudad de Punta Arenas. Con el propósito de identificar aspectos que orienten el mejoramiento de su desempeño escolar y adaptación al ambiente educativo.

\section{METODO.}

La presente investigación se basa en un enfoque cualitativo, siendo su método el estudio de caso. El estudio de casos es un método de investigación cualitativa que se ha utilizado ampliamente para comprender en profundidad la realidad social y educativa. Para Yin (1989) el estudio de caso consiste en una descripción y análisis detallados de unidades sociales o entidades educativas únicas (Guazmayán, 2004). Para Stake (1998) es el estudio de la particularidad y de la complejidad de un caso singular, para llegar a comprender su actividad en circunstancias concretas (Bisquerra, 2009). Según lo plantea Orlando Mella (2003), la búsqueda de significados a través del caso es de carácter generalmente fenomenológico, orientándose a obtener una comprensión profunda incluso adoptando una actitud de desconfianza en las anteriores descripciones y explicaciones. También plantea que, el análisis fenomenológico del caso único significa abordar el objeto de estudio, el caso, como una experiencia concreta del hombre, tan libre como se pueda de presuposiciones conceptuales. Se trata de adquirir una comprensión de la(s) estructura(as) esencial(es) del caso, sobre la base de ejemplos mentales proporcionados por la experiencia o la imaginación (Mella, 2003).

La investigación está dirigida a conocer la forma y función del juego libre de los niños y las habilidades presentes en su realización, por lo que el método de recolección de la información es a través de la observación tanto en el establecimiento educativo como en el hogar lo cual se complementó con las entrevistas semiestructuradas al tutor del niño y educadoras, para lo anterior se contó con el consentimiento informado de los padres de los niños y la cooperación de las familias educadoras y directores de los establecimientos educacionales.

Los criterios de inclusión considerados en la investigación son: niños y niñas entre 4 a 5 años que estén cursando el nivel de transición, y que no posean ningún diagnóstico previo. El criterio para los tutores es únicamente ser padre o madre del niño y vivir con él. Y en cuanto al criterio de inclusión de las educadoras de párvulos, este debe ser el educador directo del niño. 
Para la entrevista se realizó un análisis de temas, el cual es uno de los más utilizados y se ubica en el nivel superficial del discurso, remite al nivel referencial de la comunicación, lo cual permite rescatar lo más esencial e importante de cada entrevista para así luego poder realizar una comparación entre la información recogida por parte del tutor y lo observado en la realización del juego, esto permite generar puntos en común o contrarrestar la información con lo ya observado. Ruiz (2003) comenta que los métodos cualitativos, por su parte, analizan los datos mediante narraciones, viñetas y relatos cuyo ideal es la denominada por Gilbert Ryle <<Descripción Densas>, o sea, la interpretación de las interpretaciones de los sujetos que toman parte en una acción social (Ruiz, 2003).

La observación y las entrevistas se realizaron en dos contextos, estos fueron:

- Los establecimientos educacionales, considerando períodos de juego libre de los niños, para lo cual se consideraron al menos 3 oportunidades distintas.

- Y observación en los hogares de los niños, se consideraron dos visitas a lo menos.

\section{ANÁLISIS DE ESTUDIOS DE CASOS.}

Para describir el análisis de la información recopilada, se utilizó la narrativa, que permite relatar las observaciones de los distintos contextos y las entrevistas semiestrucuturadas. De los siete casos estudiados, en general se aprecia que en la mayoría de ellos la forma y función del juego no presenta mayores diferencia en los dos contextos observados, es decir casa y jardín infantil, apreciándose en ambos contextos un estilo de juego relativamente similar, colmado de las características individuales y matices de cada niño, cabe destacar que el estilo de juego elegido se condiciona y relaciona en forma importante con el acceso a distintos dispositivos y juguetes. Por otra parte se acentúa la interacción social al existir otros pares con los cuales se desarrollan actividades lúdicas interactivas. No obstante la situación es distinta en dos casos observados, donde el contexto es un factor determinante de la aproximación y elección del juego, influyendo de manera importante en la forma y función de esta ocupación. En este resumen se hará mención a dos casos que presentan mayor riqueza en la información que otorgan y representan también aspectos comunes de los datos recogidos en la totalidad de los casos estudiados.

1 Análisis de Forma y Función del juego libre del niña 1; según observación jardín infantil

Uno de los casos observados es el de una niña de 5 años, la cual fue referida por la directora del jardín al que asiste. Ella comenta que la menor no juega con "normalidad" como sus otros compañeros lo hacen. Tras un análisis minucioso, se puede inferir que el comportamiento de bajo nivel de adaptación que surge en el ambiente escolar puede deberse a aspectos emocionales, se observa que su participación social en el juego es restringido, ya que a pesar de tener las habilidades socio comunicativas suficientes para poder integrarse o participar de manera activa, no lo hace aparentemente porque el contexto no lo favorece. Tras la observación tanto en casa como en jardín, se puede inferir 
que existe significativa diferencia en ambos ambientes, ya que por un lado, en casa la menor se desenvuelve con mucha seguridad, interactuando dinámicamente con su hermana menor, realizando actividades que cumplen la función del juego como medio socializador y desarrollo de destrezas principalmente motoras (correr, andar en bicicleta, manipular pequeños objetos). Se puede observar que sólo en casa, la menor alcanza un nivel de confianza necesario para poder relacionarse y realizar una amplia gama de actividades, a diferencia de su desempeño en el jardín, donde ella se observa pasiva, incorporándose solamente a las actividades estructuradas planteadas por las educadoras, mostrando preferencia por juegos de carácter cognitivos y motricidad fina, en contraparte de los observados en su ambiente familiar. La forma del juego es indudablemente distinta en ambos contextos, y esto no solo hace referencia al entorno que abarca la realización del juego, es decir disponibilidad de espacio, implementos o juguetes, sino también se relaciona con la conducta que tiene la niña frente al juego y su entorno social. En casa el juego adquiere una connotación de carácter social- motor, siendo un personaje totalmente activo, centrada en la interacción con su hermana, mientras que en el jardín, la niña es solo una observadora pasiva de su contexto. Esta conducta reflejada en el juego como medio, hace referencia al juego de espectador, El juego de espectador es aquel en el cual un niño solo mira como otro niño juega (Berger, 2006).

En el entorno existen varias condicionantes para el juego de la niña, encontrando una directa relación entre éste y el ambiente en donde se realiza. Al parecer el factor mas influyente en la forma del juego de la niña, no se relaciona con la existencia de alguna alteración de habilidades del desempeño ocupacional o de su psicomotricidad, sino que es el contexto, el cual determina la forma y función del juego, en la Escuela esta forma del juego libre se presenta poco fluida y disarmonica, presentando su conducta más bien una función de expresar su bajo nivel de adaptación al grupo y necesidad de apoyo. Cabe destacar que esta conducta pasiva y observadora de la menor se da en períodos de juego libre al interior del establecimiento educacional, en los cuales la niña se separa del grupo y pareciera presentar algunas dificultades en sus habilidades de desempeño, o bajo nivel de interés por las actividades principalmente motoras. No obstante en actividades escolares estructuradas la niña participa sin dificultad y cumple con las tareas requeridas no observándose una disminución en su evaluación escolar de acuerdo a informe del jardín. Sin embargo en el hogar pareciera ser otra persona, juega con hermana de manera simbólica, y cambia de actividades lúdicas demostrando diversas habilidades.

De acuerdo a la observación del juego libre en el hogar se narra lo siguiente; "Respecto a las actividades lúdicas fuera del hogar, la niña juega en su antejardín, el cual es amplio, con pasto corto y abundante, y un pequeño sendero de tierra que va desde el portón hasta la entrada de la casa. Cabe mencionar que, para entrar a la cocina, la niña debe subir una escalera de tres escalones. Ella deja su bicicleta en el jardín y juega junto a su hermana menor con una caja de goma, en donde guarda la mayor parte de sus juguetes, como muñecas, platos, tenedores y cuchillos de plásticos, incluso alimentos de plásticos. Con los cuales, las niñas juegan a hacer comida, simulando ésta actividad" 
2 Análisis de Forma y Función del Juego Libre del niño; Niño 1, según observación: Casa y Jardín Infantil.

En ambos contextos el juego del menor se caracteriza por ser simbólico, donde su desempeño en el juego requiere motricidad gruesa y fina, además tiene preferencia por jugar acompañado, de tal manera interacciona activamente con sus pares y hermano menor en casa. Se destaca la función del juego como medio para interaccionar socialmente con su contexto, donde este mismo se ve representado en las distintas situaciones de juego, donde el menor realiza un juego predominantemente motor, donde su función contribuye con el desarrollo del menor. Así también existen diferencias del juego en estos contextos. Se puede mencionar que en casa se aprecia como un "buscador de sensaciones" (Imperatore 2005), esto se aprecia al observarlo mientras juega sobre los sillones de su casa, dando brincos y efectuando acciones peligrosas, como el parase de cabeza sobre los sillones, haciendo equilibrio sobre estos, pudiendo generar posibles accidentes. Donde da la impresión que este niño busca sensaciones con cambios gravitacionales y movimientos bruscos, siendo la función de este juego poder adquirir sensaciones vestibulares inconscientemente. Otra diferencia es el nivel de cognición que se puede percibir en el desempeño del juego, caracterizándose en casa por utilizar el computador como juego, con lo cual se observa motivado y lleva a cabo ciertas destrezas como, resolución de problemas, concentración, atención, comprensión. Siendo estas mas presentes mientras juega con un video juego, que el utilizar el material didáctico del jardín.

Por último se puede mencionar que a través del desempeño que presenta en el juego libre, se pudo observar diversas habilidades que sobresalen de sus pares. Y que de acuerdo a las diversas teorías del juego presenta un desarrollo de acuerdo a su edad.

De acuerdo a la observación del juego libre se narra lo siguiente: jugando en el patio de su casa... el cual tiene un arco de futbol, una bicicleta y un carro de metal a pedales. El juega con el carro, subiéndose en éste y lanzándose por la pendiente hasta donde termina el patio. El niño no presenta dificultades al momento de subirse al carro, considerando que este es un juguete que no es acorde a su contextura física, mostrando un alto nivel de destrezas para utilizarlo, ya que, resulta pequeño para él, porque le fue regalado hace un par de años atrás. Aun así, el menor al lanzarse no se cae y lo maneja sin ningún tipo de dificultad. En este juego el menor se presenta con gran energía, subiendo y bajando la pendiente una y otra vez, sin denotar gran cansancio. Se muestra alegre e interesado en el juego que está realizando. 


\section{CONCLUSIÓN}

Este estudio surgió bajo la mirada de la Ciencia de la Ocupación, donde el centro o foco de atención es la ocupación más importante de un niño, el juego, y cómo ésta ocupación como medio y fin, influye en el desarrollo habilidades y destrezas que favorecen el desarrollo integral del menor. A su vez el niño presenta comportamientos distintos en los dos ambientes observados presentando en algunos casos una conducta o estilo de juego completamente distinta en su hogar, apreciándose con esto la influencia del ambiente en la elección de las actividades del niño, como también la posibilidad de interactuar con sus pares lo cual en algunos favorece y en otros limita su participación espontánea.

Por otra parte se aprecia que los niños que poseen un juego libre más fluido e imaginativo presentan también mejores resultados en sus pautas de evaluación escolar.

En esta investigación se realizó el estudio de 7 casos de niños de pre básica, entre 4 y 5 años, observando y analizando su ocupación de juego en contextos de su casa y jardín en los que se analizó la forma y función del juego libre. Donde se pretendió abarcar toda la particularidad y complejidad de la misma, de tal manera llegar a poder realizar las siguientes conclusiones:

- El juego es el medio por el cual los niños son capaces de desarrollar distintas habilidades, las cuales influyen en su desempeño preescolar.

- La función del juego adquiere distintas connotaciones, en general sirve para desarrollar destrezas motoras, sociales, cognitivas, emocionales y sensoriales, las cuales en equilibrio favorecen el desarrollo integral del menor.

- Tras la observación de la forma y función del juego libre del niño, se destaca el juego principalmente como medio para desarrollar la interacción social.

- A raíz de la forma de esta ocupación, se puede detectar ciertos, rasgos, falencias, disfunciones, que influyen en la manera de como juega el menor y lleva a cabo su realización.

- En general el contexto se puede presentar como un facilitador o una barrera en la forma y ejecución del juego libre del menor, debido a que puede proporcionar oportunidades o instancias de estimulación en los más diversos entornos y otorgar posibilidades de interactuar con otros, ya sea compañeros o familia. De tal manera sus características particulares pueden influir en su desempeño ocupacional y la manera en que el menor se adapta a su contexto físico- social.

- Un factor primordial que influye en la realización del juego del menor es, el factor clima, este delimita la ejecución libre del juego, considerando las condiciones regionales, las 
bajas temperaturas, precipitaciones, los fuertes vientos, y la humedad, entre otros, limitan la posibilidad de realizar un juego al aire libre.

\section{DISCUSIÓN Y REFLEXIONES.}

En relación al objetivo de describir la forma de la ocupación del juego libre del niño, se puede destacar que su forma es altamente influenciada por el contexto donde se desenvuelven los niños, ya que este contiene características particulares que hacen posible su realización, los implementos y aspectos que posee este contexto físico- social. Por ejemplo, si en el salón del jardín hay determinados juguetes el niño intentara adaptarse al contexto y realizara un juego direccionado por este entorno físico y social que lo contiene. En relación al segundo objetivo de identificar la función del juego libre de los niños, se puede mencionar que su función, que puede asociarse al desarrollo de destrezas o la adaptación social entre otras, parece ser altamente influenciada por el contexto y las oportunidades o barreras que este proporciona. Sin embargo cabe destacar que en general la esencia del niño, su preferencia en los juegos y en el estilo de actividades seleccionadas se mantiene, sólo cambia la manera en que se realiza el juego o los elementos de los que dispone. Por ejemplo, al observar a los niños en ambos contextos en la mayoría de los casos, la función social, motriz, intelectual sigue predominando de acuerdo a sus características individuales, el menor que juega en jardín con sus compañeros, juega en casa con familiares, la función de interactuar con su entorno social no varía, si el menor se dedica a jugar en el jardín infantil con autos, puzles, correr jugando al pillarse, en casa utiliza otros juguetes articulados, legos, bicicleta, la función de desarrollar o fomentar la motricidad no varía, sólo cambia la manera, la forma y el medio para realizarlo.

A diferencia de lo anterior, los dos casos que se desarrollaron más en detalle en los puntos 3.1 y 3.2, tienen en común la diferencia en que ambos niños juegan e interactúan en situación de juego libre en los dos ambientes observados, estos son casa y jardín infantil, considerando que ambos contextos presentan opciones relativamente similares de opciones para la interacción con otros pares y acceso a diversos estímulos o juguetes. Dejando planteada la inquietud de porqué estos niños teniendo las habilidades, destrezas e interés en participar en los diversos tipos de juego, presentan distinta preferencia y aproximación a las actividades lúdicas de manera espontánea en distintos contextos, llegando a parecer que presentaran deficiencia en algunas funciones e incidiendo finalmente en su desempeño y evaluación escolar.

\section{SUGERENCIAS.}

A futuro se visualiza la posibilidad de:

- Desarrollar futuras investigaciones de tipo clínico, respecto al juego del niño preescolar, para así poder detectar a tiempo ciertos patrones de conducta desadaptativas con el objetivo de que ciertas alteraciones o disfunciones, sean tratadas y que a futuro su desempeño ocupacional no se vea afectado. 
- Dar continuidad a la investigación, analizar la forma y función del juego libre del niño, en etapa escolar y así poder analizar diferencias de su ejecución y tiempo empleado, haciendo referencia a nuevos factores como por ejemplo organización y temporalidad.

- Realizar futuras aproximaciones en relación a la influencia de factores climáticos sobre la forma y función de éste dentro del contexto.

\section{REFERENCIAS BBLIOGRÁFICAS}

Berger, K, (2006). Psicología del desarrollo: Infancia y Adolescencia. Estados Unidos. 7ma Edición. Panamericana.

Bisquerra, R. (2009). Metodología de la investigación educativa. España. 2da edición. La Muralla, S.A.

Christiansen, Ch. Defining lives: Occupation as identity : An essay on competence, coherence, and creation of meaning. AJOT, 1999.

Guazmayán, C. (2004). Internet y la investigación científica: El uso de los medios y las nuevas tecnologías en la educación. Colombia, 1ra Edición, Alma Mater.

Imperatore Blanche, Erna. (2005)"Déficit de Integración Sensorial: Efectos a largo plazo sobre la ocupación y el juego". Revista Chilena de Terapia Ocupacional № 5.

Mella, O, (2003), Metodología Cualitativa en Ciencias Sociales y Educación: Orientaciones Teórico-Metodológicas y Técnicas de Investigación, Santiago, Chile.

Patricia M. Manuel (2003) Occupied with ponds: exploring the meaning, bewaring the loss for kids and communities of nature's small spaces. Journal of Occupational Science, Vol 10.

Polonio, B. (2008). Terapia Ocupacional en la infancia Teoría y práctica. España, 1ra Edición, Panamericana.

Ruiz, J. (2003). Metodología de la investigación cualitativa. España. 3ra Edición.

Willard, H, Crepeau, E y col (2005) Terapia Ocupacional, España, 10 edición, editorial Panamericana 